\title{
Interaction and vectoring of parallel rectangular twin jets in a turbulent boundary layer
}

\author{
Girish K. Janke ${ }^{*}$ and Bharathram Ganapathisubraman $\dagger^{\dagger}$ \\ Aerodyamics and Flight Mechanics, \\ Faculty of Engineering and Physical Sciences, \\ University of Southampton, SO17 1BJ, \\ UK
}

(Dated: March 18, 2021)

\begin{abstract}
The ability to impart momentum while maintaining a zero net mass flux renders synthetic jet actuators attractive tools for a wide variety of applications. Implementation of a single synthetic jet actuator for large-scale operations is unrealistic and as such, an array of actuators is usually desired during flow control processes. The added complexity of several synthetic jets in close proximity and the subsequent jet-jet interaction, in addition to interaction with the crossflow, represent an area of research yet to be fully explored. This paper encompasses a parametric study to investigate the interaction of a zero pressure gradient turbulent boundary layer $\left(R e_{\tau}=1300\right)$ with twin parallel synthetic jets, where the major axis of the rectangular orifices is aligned with the crossflow. Only the separation distance, $s$, and the phase difference, $\beta$, between the two orifices are varied. Geometrical parameters such as the orifice shape and aspect ratio $(A R=13)$, as well as fluidic properties such as the jet Strouhal number $(S t=2.3)$ and the momentum coefficient $\left(C_{\mu}=0.16\right)$ are kept constant throughout. Velocity fields acquired through stereoscopic PIV measurements at 5 downstream locations indicate noticeable differences in the flowfield and associated stresses. A limit in spacing is noted beyond which any subsequent increase results in the twin jets behaving as two independent synthetic jets. In comparison to a single synthetic jet in crossflow, the results demonstrate that twin jets operated at a specific phase difference and spacing can be equally, if not, more efficient for flow entrainment and momentum distribution.
\end{abstract}

\section{INTRODUCTION}

Over the past decades, synthetic jet actuators (SJAs) have emerged as key devices in various applications pertaining to fluid mechanics. Whether in flow control domains or in enhancing the cooling efficiency of thermal systems, the rich and complex structures belonging to the counter rotating vortex pair (CVP) remain of particular interest to researchers. Numerous investigations have characterised the underlying principles governing the formation and evolution of synthetic jets both in quiescent conditions (Glezer and Amitay, 2002, Holman et al. 2005, Smith and Glezer, 1998) and under the influence of a crossflow (Berk and Ganapathisubramani, 2019, Jabbal and Zhong, 2008, 2010, Smith, 2002, Van Buren et al., 2016a b). Although most studies focus on a single synthetic jet actuator, the use of a single actuator in most real-world applications is unrealistic and instead an array consisting of several actuators is often required.

For example, multiple actuators need to be fitted over the entire span of an aircraft wing or rudder when attempting to delay flow separation or to control the large scale structures in a turbulent wall-bounded flow to reduce skin-friction drag. In spite of such pertinence, lim-

\footnotetext{
* girish.k.jankee@ntnu.no

$\dagger$ G.Bharath@soton.ac.uk
}

ited literature is available on two or more parallel jets in a crossflow. There has been extensive research carried out in understanding the interaction of parallel twin jets in quiescent flow (Berk et al. 2016 , Luo and Xia, 2008; Smith and Glezer, 2005) and twin jets arranged in an in-line configuration in a crossflow, i.e., the orifices are placed one behind another, in a linear pattern (Honami and Motosuke, 2012, Wen et al., 2015, 2016, Yu et al., 2006). However, the added complexity of parallel jetjet interactions with a crossflow is still unexplored in a detailed and systematic parametric approach.

\section{A. Twin jets in quiescent flow}

In the absence of a crossflow, two key parameters dictate the interaction and evolution of parallel synthetic jets: (i) the spacing between the orifices, $s$, and (ii) the phase difference between the jets, $\beta$ (Berk et al. 2016. Luo and Xia, 2008; Smith and Glezer, 2005; Watson et al. 2003). In such a configuration, Luo and Xia (2008) found that the interaction between the jets lead to two types of mechanism based on the initial operating conditions, namely, an enhancing mechanism and a vectoring mechanism (Fig. 11). Similarly, Berk et al. (2016) experimentally observed that for two orifices close enough, the resulting jet from the interaction process can either be merged, bifurcated or vectored towards the actuator 


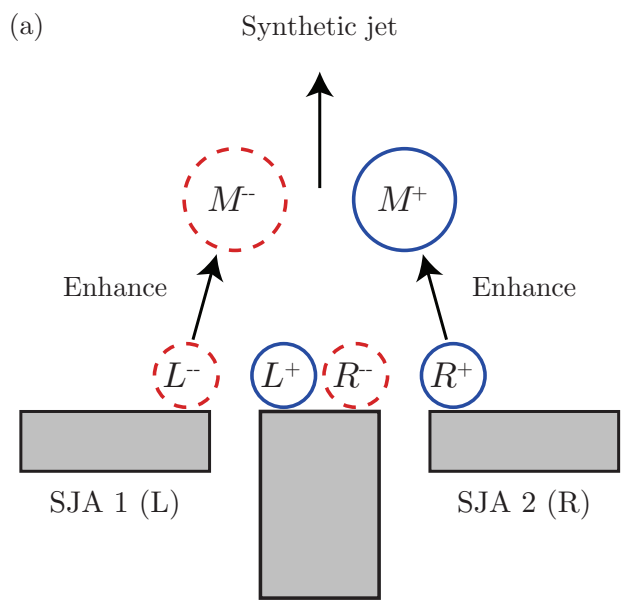

(b)

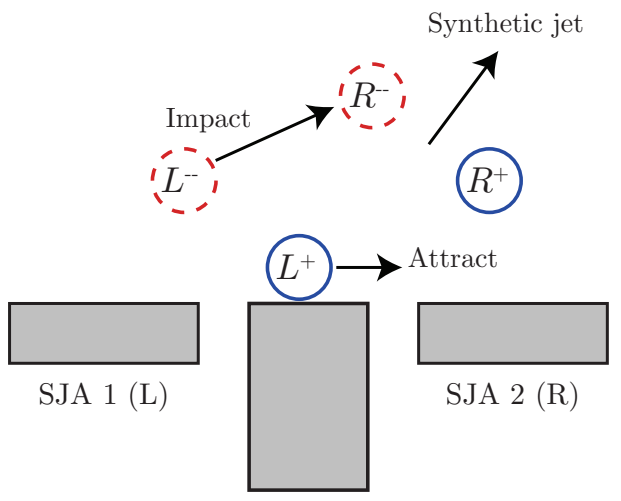

FIG. 1 Interactions between parallel synthetic jet actuators in quiescent flow, adapted from Luo and Xia (2008) and Berk et al. (2016). (a) Enhancement mechanism which occurs in the absence of a phase difference between the two jets and (b) Vectoring mechanism associated with the introduction of a phase difference.

which is leading or lagging in phase.

When there is no phase difference $(\beta=0 \mathrm{deg})$, an enhancing mechanism, sensitive to the non-dimensional separation distance, $s / d$, occurs. The merged synthetic jet has similar properties to a single synthetic jet with the same centerline velocity but achieves a much higher amount of entrained fluid (Smith and Glezer, 2005). As sketched in Fig. 1a, the rolling-up of the clockwise $\left(L^{+}\right)$ vortex from SJA1 and the counterclockwise $\left(R^{-}\right)$vortex from SJA2 results in a induced velocity acting on the opposite ring due to the oppositely sign vortices cancelling out each other (Watson et al. 2003). Futhermore, the pressure in the region of the vortex pair is substantially reduced and coupled with this induced velocity, causes the synthetic jets from each actuator to modify their trajectory and attract each other, eventually coalescing into a single enhanced vortex pair (Luo and Xia, 2008). The strength of this enhancing mechanism abates with increasing $s / d$. For $s / d$ larger than 6 , Watson et al. (2003) noted that vortex rings from adjacent orifices advect downstream without any perturbations. This is corroborated by McGuinn et al. (2016) who used an array of actuators for heat transfer enhancement and found no noticeable change in the temperature distribution for $s / d$ of 6 and above.

The introduction of a phase difference between the jets $(\beta>0 \mathrm{deg})$ results in a vectoring mechanism. Considering the reliance of vectoring on the interaction between the two jets, it can be inferred that vectoring only occurs when $s / d$ is less than the threshold value of 6 observed by Watson et al. (2003) and McGuinn et al. (2016). Luo and Xia (2008) described vectoring as a combination of attraction and impact between vortices which results in the deflection of the resulting jet. As the phase angle increases, one jet leads during the blowing cycle. Since the leading jet reaches peak blowing first, it generates a momentum flux to the surrounding flow due to its CVP. As the lagging jet starts to blow, the low pressure in the wake of the fore vortex pair attracts the CVP generated by the lagging jet. Further, since the leading vortex pair is slowed down through a loss of energy due to friction and entrainment of surrounding fluid, the lagging CVP impacts with the leading one and a merged jet, vectored in the direction of the actuator leading in phase emerges (Fig. 1p). Berk et al. (2016) observed that the vectoring angle, $\gamma$, increased with phase difference up to the point where the merged jet becomes fully attached to the surface. When $\beta$ is increased such that the jets are completely out of phase, a reduction in vectoring and flow rate is observed since the fluid ejected from one orifice is ingested into the adjacent orifice (Luo and Xia, 2008).

\section{B. Twin jets in crossflow}

The limited amount of literature available for twin parallel rectangular synthetic jets in crossflow convolutes the interpretation of structures present in the flowfield in such a configuration. The interactions between parallel synthetic jets emanating from circular orifices and a crossflow was investigated comprehensively by Zang and New (2017). The results showed that each jet in a twin jet configuration was capable of achieving higher entrainment and jet half-widths as opposed to a single jet in crossflow. A reduction in $s / d$ resulted in the twin jets interacting with each other closer to the orifice exit. Similar to the enhancement mechanism in quiescent flow, the inner vortices were observed to move towards each other, neutralise each other due to the oppositely signed vorticity and coalesce into a single CVP, which prevailed downstream. These results are consistent with the findings of Toy et al. (1993) and large eddy simulation (LES) by Schlüter and Schönfeld (2000), which showed the emer- 
gence of a single CVP for $s / d$ of 5 . Zang and New (2017) found the interaction between the two parallel jets to be more prominent at low velocity ratios. Such a trend occurs since at lower velocity ratios, the CVPs are weaker and hence, more sensitive to mutual interactions. Additionally, Zang and New (2017) attributed the early interactions between the jets to the jet cores being deflected over a shorter downstream distance. A consequence of jet-jet interaction is an increase in the Reynolds shear stress and enhancement in the levels of entrained fluid in regions adjacent to the centerline plane between the two parallel orifices.

In addition to the number of SJAs in the array and $s / d$, Holdeman (1993) and Orkwis and Filz (2003) highlighted the importance of the momentum ratio in jet penetration and mixing. Numerical simulations established that the momentum imparted to the crossflow is sensitive to the spacing between the two orifices. Based on Jankee and Ganapathisubramani (2021), the momentum ratio between the jet issued from a rectangular orifice and crossflow is defined as the momentum coefficient, $C_{\mu}$ :

$$
C_{\mu}=\frac{\rho \bar{u}_{j}^{2} l d}{\rho u_{\infty}^{2} \theta d}=r^{2} \frac{l}{\theta},
$$

where $\overline{u_{j}}$ is the mean jet exit velocity, $u_{\infty}$ is the freestream velocity of the crossflow, $l$ is the length of the orifice aligned with the flow and $d$ is the orifice width in the cross-stream direction. $\theta$ is the momentum thickness of the boundary layer and is used as it characterises the boundary layer's ability to overcome the adverse pressure gradient due to the jet. For small $s / d$, twin synthetic jets provide a lower momentum than a single jet while this trend is reversed for large $s / d$. For specific operating conditions (phase difference, actuation frequency and mean blowing velocity) and orifice spacing, Orkwis and Filz (2003) found twin jets to have a net momentum contribution of $6 \%$ more than two individual SJAs. Furthermore, Hasnain et al. (2012) observed the recirculation zone shape to change depending on the phase difference between the jets and the absence/presence of a crossflow. Hasnain et al. (2012) also noted the vectoring behaviour in crossflow to be similar to quiescent flow conditions. However, it was concluded that the dynamics of twin jets in crossflow cannot be simplified to a linear superposition of the dynamics of two individual single jets in crossflow.

Although there has been progress towards understanding the evolution of twin parallel synthetic jets in crossflow, these have primarily focused on circular orifices and as such, the relevance of these findings to rectangular orifices remain equivocal. The aim of the present study is to understand the dynamics associated with parallel twin synthetic jets emanating from rectangular orifices and their subsequent interaction with an incoming turbulent boundary layer. By varying physical parameters such as the spacing between the orifices and the phase difference between the jets, this investigation aims to characterise and quantify the sensitivity of the enhancing and vectoring mechanisms and the associated shear stresses on these variables.

\section{EXPERIMENTAL SETUP}

\section{A. Actuator design}

The synthetic jet actuator used in this study consists of three main components: the driver (a loudspeaker), a cavity assembly and the orifice. To achieve a twin jet configuration, a second actuator is positioned adjacent to the first one, with the major axis aligned parallel to the incoming flow (Fig. 2(a)). Both actuators have a pancakeshaped cavity with a volume, $V_{c}$ of $2.63056 \times 10^{-5} \mathrm{~m}^{3}$. The pancake-shaped cavity is important as it allows the orifices to be positioned close together. The geometrical properties of the actuators, including the aspect ratio $(A R=l / d)$ of the orifices, are kept constant throughout the study. The orifices have a rectangular slot with a fixed width, $d$ of $1 \mathrm{~mm}$ and fixed length, $l$ of $13 \mathrm{~mm}$, resulting in an aspect ratio, $A R$, of 13 . The SJAs are driven independently, each with a Visaton SC $8 \mathrm{~N}$ speaker with an impedance of $8 \Omega$, a rated power of $30 \mathrm{~W}$, a frequency response between 70 and $20000 \mathrm{~Hz}$ and diaphragm resonance occurring around $110 \mathrm{~Hz}$. The test cases combining a range of orifice spacing and phase differences between the jets are detailed in Table [

TABLE I The combination orifice spacing and phase difference between the jets resulted in 18 differing test configurations. The velocity ratio, $r$, Strouhal number, $S t$ and $A R$ of the rectangular orifices are maintained at $0.88,2.3$ and 13 , respectively, for all test cases.

\begin{tabular}{cc}
\hline \hline$s / d$ & $2,6.5,13$ \\
$\beta($ deg $)$ & $0,12,78,108,138,168$ \\
\hline \hline
\end{tabular}

\section{B. Description of facility}

The experiment was carried out in a suction wind tunnel facility at the University of Southampton. The test section has a length of $4.5 \mathrm{~m}$, width of $0.9 \mathrm{~m}$ and a height corresponding to $0.6 \mathrm{~m}$. A false floor made up of smooth Aluminium plates is fitted in order to generate a zero pressure gradient turbulent boundary layer. The false floor has a sharp leading edge with a zigzag trip $0.03 \mathrm{~m}$ downstream and an adjustable flap to control the position of the stagnation point at the leading edge. The two SJAs are embedded in the false floor such that the major axis of the orifices is aligned with the incoming flow and is flushed with the surrounding surface (Fig. 3a). 
(a)

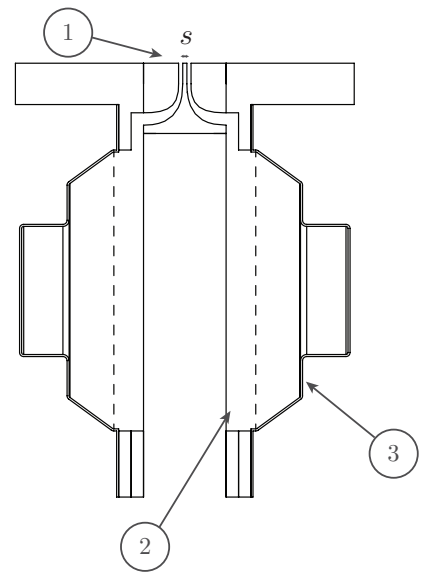

(b)
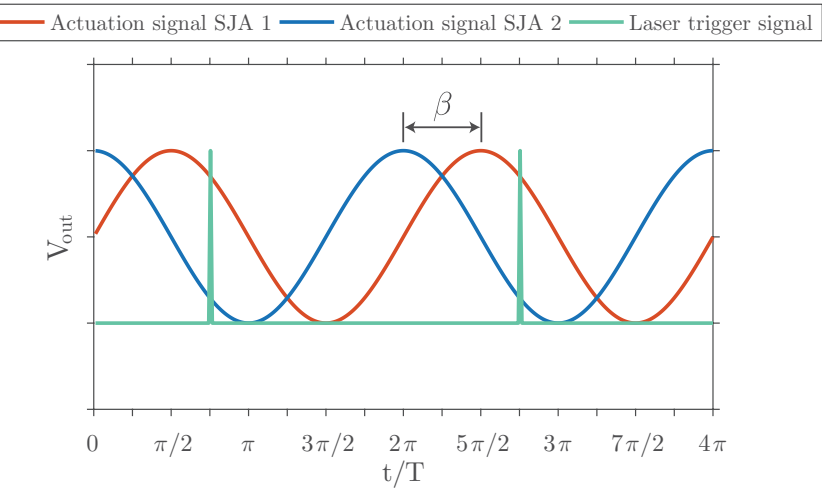

FIG. 2 (a) Schematic of synthetic jet actuator design in parallel twin-jet configuration (1. Orifice exit, 2. Cavity assembly and 3. Loudspeaker). (b) Twin jet actuation signal with applied phase difference and phase-locking trigger signal.

The freestream velocity was kept constant at $10 \mathrm{~m} / \mathrm{s}$ for all test cases. This resulted in a turbulent boundary layer with thickness, $\delta$, of $45 \mathrm{~mm}$ based on $U(\delta)=U_{99}$ (Fig. 33). Using typical values of $\kappa=0.384$ and $\mathrm{A}=4.4$ from law of the wall analysis, results in a friction velocity of $0.38 \mathrm{~m} / \mathrm{s}$. This results in a flow Reynolds number, $R e_{\tau}$, of 1300 . Normalising the region of interest with the boundary layer thickness culminates in a flowfield which spans from $\mathrm{x} / \delta=-2.5$ to $\mathrm{x} / \delta=2.5$ in the spanwise direction and up to $y / \delta=1.5$ in the wall-normal direction. This provides sufficient area to capture the interaction of both jets with the crossflow.

\section{Hot wire anemometry}

The actuators are calibrated and characterised using hot-wire anemometry under quiescent conditions. A DANTEC Streamline Pro CTA system with two singlewire traversing hot-wire probes with a diameter, $d_{w}$, of $2.5 \mu \mathrm{m}$ and prong spacing of $2.5 \mathrm{~mm}$ are operated in constant temperature mode with an overheat ratio of 0.8 . The wire resistance is measured to be $4.5 \Omega$. The probes are aligned such that the sensing bit is positioned at the center of the orifice exit for each SJA and placed at a height of $1 d$, away from the orifice exit. The hot-wire signal was sampled for 30 seconds at a sampling rate of $35 \mathrm{kHz}$ and low-pass filtered at $10 \mathrm{kHz}$ to remove high frequencies due to noise and prevent aliasing. The exit velocity of both jets is measured simultaneously and the mean blowing velocity of the jets is obtained using Eqn. 2 and 3 . Following rectification of the signal from the probe, the velocity of the synthetic jet can be expressed as:

$$
u_{0}(t)=u_{\max } \sin \left(\frac{2 \pi t}{T}\right)
$$

where $u_{0}(\mathrm{~m} / \mathrm{s}), u_{\max }(\mathrm{m} / \mathrm{s}), t(\mathrm{~s})$ and $T(\mathrm{~s})$ represent the variation in jet exit velocity over time, the maximum velocity during the blowing phase, the time and period of the cycle, respectively. Based on Smith and Glezer (1998), the mean blowing velocity $(\bar{u})$ can then be derived as follows:

$$
\bar{u}=\frac{1}{T} \int_{0}^{T / 2} u_{0}(t) d t=\frac{u_{\max }}{\pi} .
$$

The mean blowing velocity, $\bar{u}$, and the Strouhal number, $S t$, of both jets were maintained at $8.8 \mathrm{~m} / \mathrm{s}$ and 2.3 respectively, throughout the experiments.

\section{Stereoscopic PIV}

Cross-stream stereoscopic PIV (SPIV) measurements were performed in the $y-z$ plane at 5 different streamwise locations, namely $x / \delta$ of $0,0.5,1,3$ and 6 . Two aligned Litron $200 \mathrm{~mJ}$ dual pulse Nd-YAG lasers were used to create a single laser sheet with a thickness of $1 \mathrm{~mm}$ while seeding is provided by a Martin Magnum 1200 machine, ejecting particles with a mean physical diameter of $1 \mu \mathrm{m}$. Two Lavision Imager Pro LX 16MP cameras, each fitted with a $300 \mathrm{~mm} \mathrm{f} / 8$ Nikon lens, were placed on either side of the wind tunnel and locked at an angle, $\theta$ corresponding to $35 \mathrm{deg}$ with respect to the region of interest (Fig. 3a).

The laser and the cameras were phase-locked to the driving signal of the actuator leading in phase at 8 equidistant phases $(\phi)$ of the actuation cycle. For each phase, 200 image pairs per camera were recorded. Vector fields were determined by GPU processing using Lavision Davis 8.3.0 software. An initial step with a window size of $64 \times 64$ px with an overlap of $50 \%$ was applied followed by two passes of $32 \times 32$ px with a $75 \%$ overlap. This resulted in a resolution of $0.25 \mathrm{~mm}$ per vector in both the $y$ and $z$ directions. Bhattacharya et al. (2017) defined 
(a)

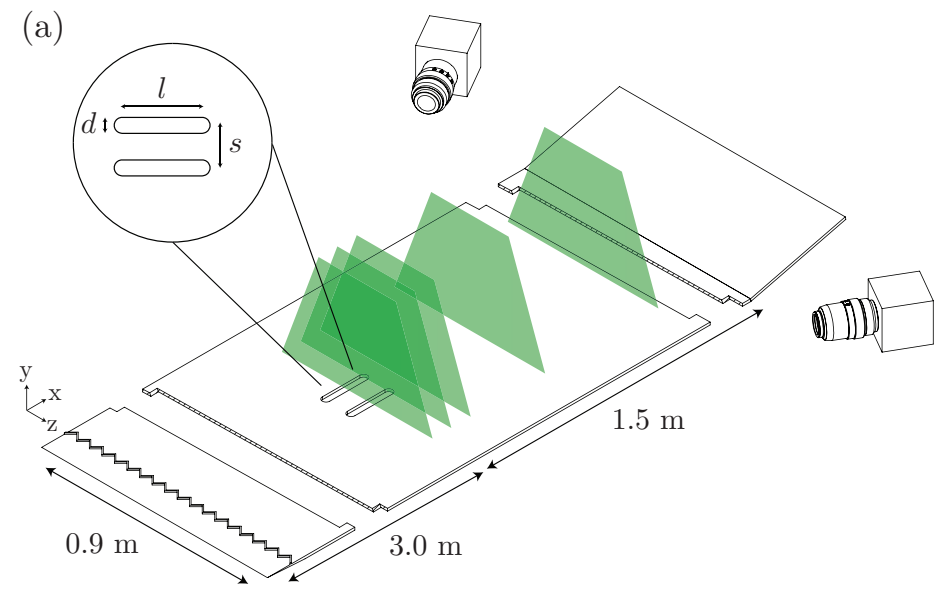

(b)

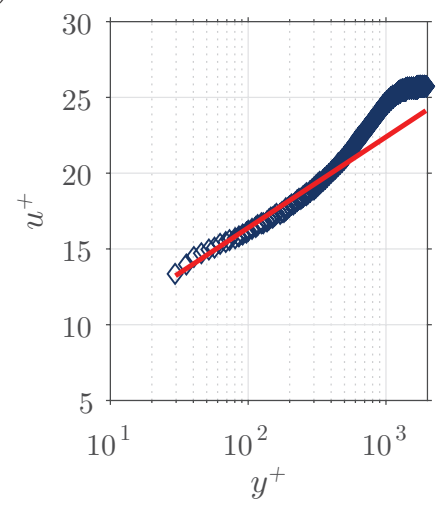

FIG. 3 A schematic of the test facility is presented. (a) A view of the wind tunnel configuration with a zigzag trip at the leading edge, the major axis of the orifices aligned with the incoming flow, a flap at the trailing edge to control the position of the stagnation point and laser sheets and cameras for SPIV measurements (not to scale). (b) Spanwise averaged boundary layer profile of the unperturbed flow at $x / \delta=0$ (Blue diamond) compared to a log law with coefficients $\kappa=0.384$ and $B=4.4$ (red line). This work follows the convention that $x, y$ and $z$ are the streamwise, wall-normal and spanwise directions with $U, V$ and $W$ being the corresponding velocities in those directions.

a methodology to quantify the uncertainty in SPIV and found that planar PIV uncertainty for each camera and uncertainty in the stereo calibration function were main contributors. Beresh et al. (2016) also explained that self-calibration techniques might not necessarily eliminate bias errors such as pixel locking for large stereoscopic angles, thick laser sheets and small particle diameters. Jankee and Ganapathisubramani (2020) examined the latter and provided a potential a posteriori technique to mitigate the influence of pixel locking in SPIV measurements. Taking into account these factors and following the methodology from Bhattacharya et al. (2017), calculations showed a $1.5 \%$ bias uncertainty in the velocity measurements for an average particle displacement of 7 pixels, considering that the sub-pixel uncertainty is approximately \pm 0.1 pixel.

\section{RESULTS AND DISCUSSION}

\section{A. Mean flowfield}

Analysis of the near field velocity components reveals the structures and intensity of interactions between the jets themselves and with the crossflow. Fig. 4 displays the evolution of the twin jets through the blowing part of the actuation cycle $(\phi=0-135 \mathrm{deg})$ when there is no phase difference between the jets $(\beta=0 \mathrm{deg})$. Contour plots of the streamwise boundary layer subtracted velocity component in the near field $(x / \delta=0)$ are shown as the orifice spacing $(s / d)$ is varied. Vortical structures are identified using the vorticity-signed swirling strength $\left(\lambda_{c i}\right)$ (Fig. 5). Following Ganapathisubramani et al. (2002), the swirling strength is obtained by multiplying the imaginary part of the complex eigenvalue of the crossplane velocity gradient tensor by the sign of the vorticity. This quantity isolates the coherent swirling structures and allows the rotation sense of the streamwise vorticity to be identified. In this study, the vorticity-signed swirling strength is normalised by the boundary layer thickness and the freestream velocity in the streamwise direction.

For $s / d=2$, the jets start to interact with each other at the onset of the actuation cycle. The inner vortex of each CVP cancels out each other due to the opposite sign vorticity. The remaining part of the vortices coalesce into a single resultant CVP evolving throughout the blowing phase, similar to the observations of Watson et al. (2003) and Luo and Xia (2008) under quiescent conditions (Fig. $4 \mathrm{a}-4 \mathrm{~d}$ ). The enhancement mechanism still perpetuates under crossflow conditions with the velocity deficit reaching twice that of a single independent jet in crossflow. This is consistent with the findings of Smith and Glezer (2005), who observed the enhancement mechanism to yield more than double the volume flow rate of entrained fluid on either side of the merged jet. Further, as observed from Fig. 4 and 5 , the crossflow has two implications. Firstly, the depth of penetration of the merged jet is less than an independent jet under quiescent conditions. This occurs because as the jets are generated and penetrate the boundary layer, entrainment effects and an adverse pressure gradient (APG) force the jets to be skewed in the direction parallel to the crossflow. Secondly, the emergence of the jets causes blockage of the incoming flow and gives rise to a horseshoe vortex, with legs visible on the sides of the primary vortex pair in Fig. 5.

As the spacing between the orifices increases to 6.5, the 


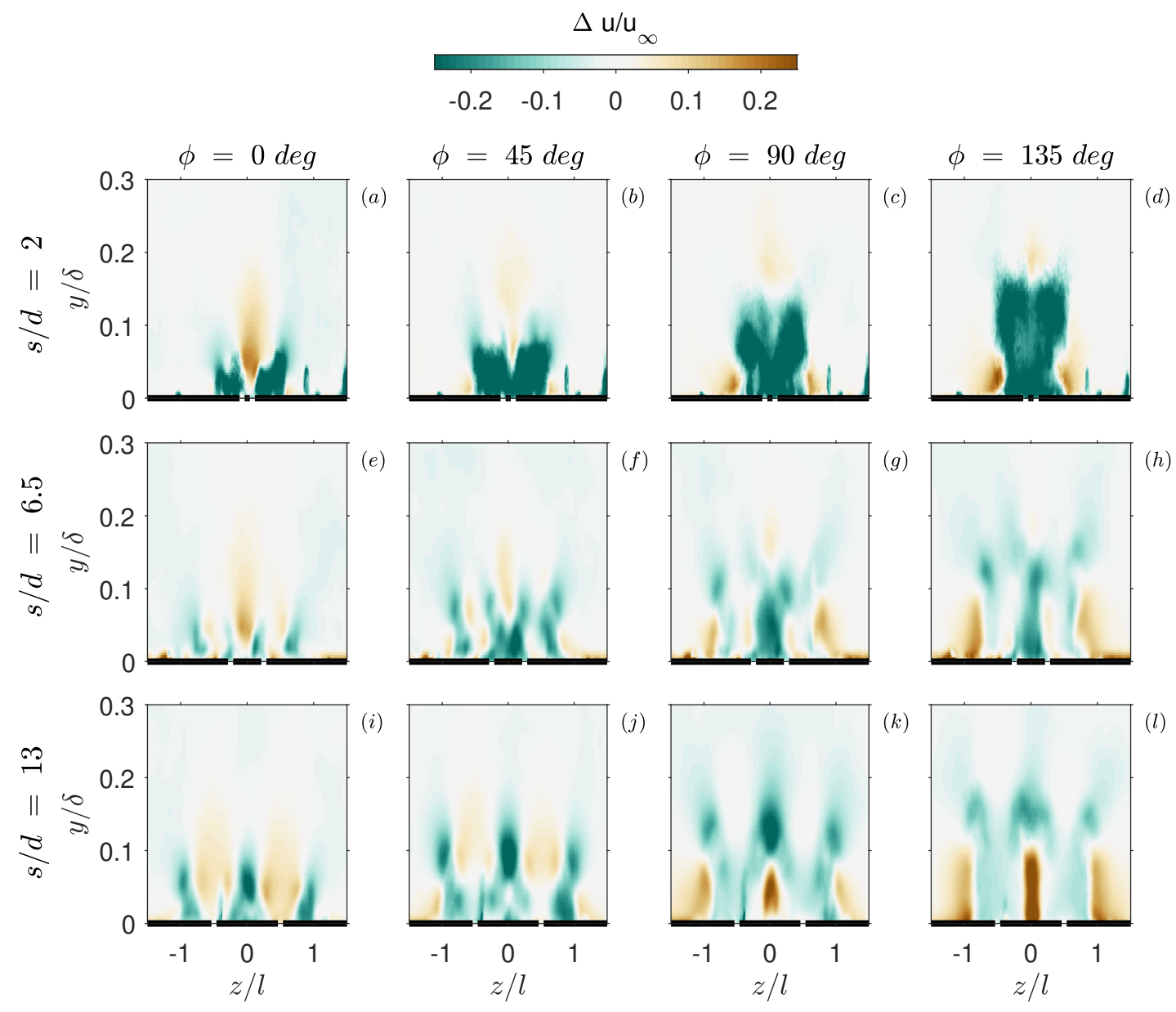

FIG. 4 Boundary layer-subtracted streamwise velocity contour plots for twin jets evolving during the blowing part of the actuation cycle for $s / d=2(\mathrm{a})-(\mathrm{d}), s / d=6.5(\mathrm{e})-(\mathrm{h})$ and $s / d=13$ (i)-(l).

degree of interaction between the two jets reduces and the strength of the enhancement mechanism is significantly diminished. At $s / d=13$, two independent jets can be observed to evolve through the actuation cycle with little or no interaction between them. When sufficiently far apart, each jet induces its own horseshoe vortex. Further, since the inner parts of the two CVPs do not annihilate each other, they create a downwash effect which causes high momentum fluid from the freestream portion of the boundary layer to move close to the wall. This is evidenced by the positive $\Delta u$ values observed between both jets (Fig. 4k and 4).

\section{B. Vectoring mechanism}

In this section, the influence of two key parameters, the separation distance between the orifices $(s / d)$ and the phase difference between the jets $(\beta)$, on the vectoring mechanism is discussed. In all figures presented henceforth, the jet on the left is always leading in phase, unless otherwise mentioned. Fig. 6 6 compares the vector- ing behaviour between $s / d$ of 2 and 13 at $x / \delta=0$. For large separation distances such as $s / d=13$, it can be observed that the two jets evolve independently of each other throughout the blowing phase. Although under the influence of a crossflow, this result is consistent with the findings of Watson et al. (2003) and McGuinn et al. (2016), which showed jets to advect without any perturbations when far apart, under quiescent conditions. However, as $s / d$ is reduced to 2 , the resulting jet experiences some inclination towards the actuator leading in phase as the blowing cycle continues. It should be noted at this point that, for all cases investigated, vectoring is only observed for $s / d=2$ and any subsequent discussion pertaining to this phenomenon only considers this specific orifice spacing.

The inclination of the resulting jet is attributed to the same vectoring mechanism as observed in quiescent flow by Smith and Glezer (2005) and Luo and Xia (2008). As shown in Fig. 6a-6 $\mathrm{d}$, the vortex pair of the lagging jet is heavily influenced by the CVP originating from the jet leading in phase. At $\phi=0 \mathrm{deg}$, only the leading jet is blowing and structures similar to a single jet in 


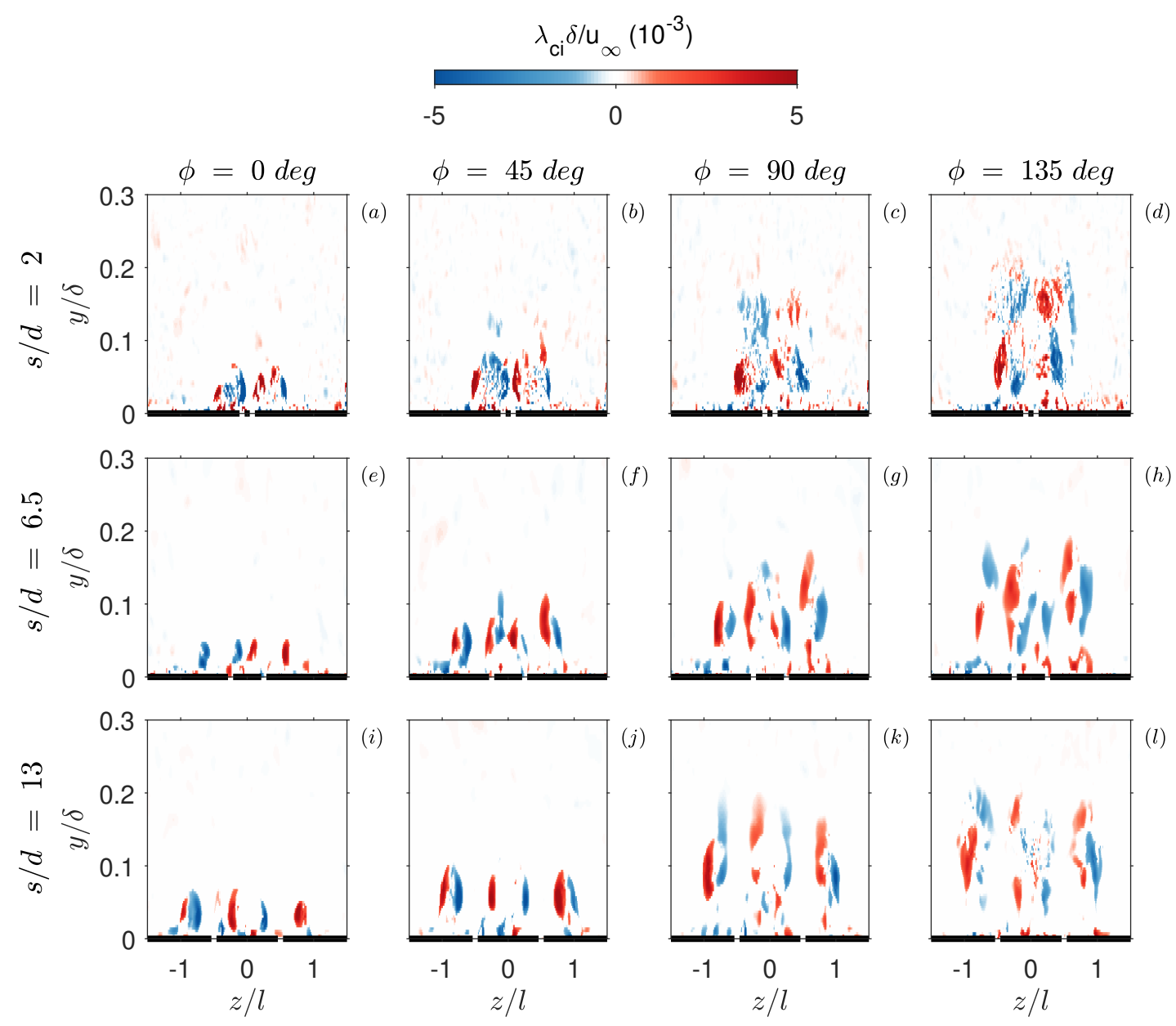

FIG. 5 Vorticity-signed swirling strength contour plots for twin jets evolving during the blowing part of the actuation cycle for $s / d=2(\mathrm{a})-(\mathrm{d}), s / d=6.5(\mathrm{e})-(\mathrm{h})$ and $s / d=13(\mathrm{i})-(\mathrm{l})$, all at $\beta$ of $0 \mathrm{deg}$.

crossflow can be observed, viz., a CVP and a horseshoe vortex on the sides. The horseshoe vortex is a permanent feature of the jet in crossflow due to blockage caused by the jet and prevails throughout the blowing part of the actuation cycle. As $\phi$ increases, part of the CVP from the leading jet convects downstream while the nearfield features are influenced by the blowing cycle of the lagging jet. The inner legs cancel each other out due to oppositely signed vorticity while the low pressure in the wake of the leading jet causes the clockwise $(\mathrm{CW})$ rotating vortex of the lagging jet to be attracted towards the jet leading in phase. Hence, the resulting jet being vectored towards the left.

The introduction of a crossflow impacts the vectoring behaviour in the near field of the orifices. As the structures emanating from the orifices move further downstream, increased interaction between the jets lead to two major observations. Firstly, the vectoring angle, $\gamma$, between the jets increases and secondly, the intensity of the time-averaged vorticity decreases. The effect on the vectoring angle follows the same explanation as Luo and Xia (2008) where a combination of attraction and impact changes the direction of the resulting jet. Such interaction also leads to rapid breakdown of the structures which are no longer coherent within a few boundary layer thicknesses away from the orifice exit. To achieve a better understanding of the vectoring mechanism, it is necessary to quantify the vectoring angle arising from the interaction of twin jets between themselves and with the crossflow. In this study, the protocol described by Berk et al. (2016) is adopted. The vectoring angle is computed by taking into account the velocity field, following subtraction of velocities in the flowfield when there is no actuation, in both the $y$ and $z$ direction (Eqn. 4). Further, under the influence of a crossflow, the inclination angle is not enough to describe vectoring. Rather, the spanwise offset of the center of the resulting jet, henceforth defined as the yaw offset $\left(\chi_{z}\right)$, needs to be accounted for. The yaw offset represents the spanwise distance of the center of the resulting jet from the origin. Two half circles are drawn for a distance of $r / d=5$ and $r / d=10$ from the origin, corresponding to the center-line between the two orifices at $y / \delta=0$. The use of a half-circle eliminates any bias which might be present in the vertical or 


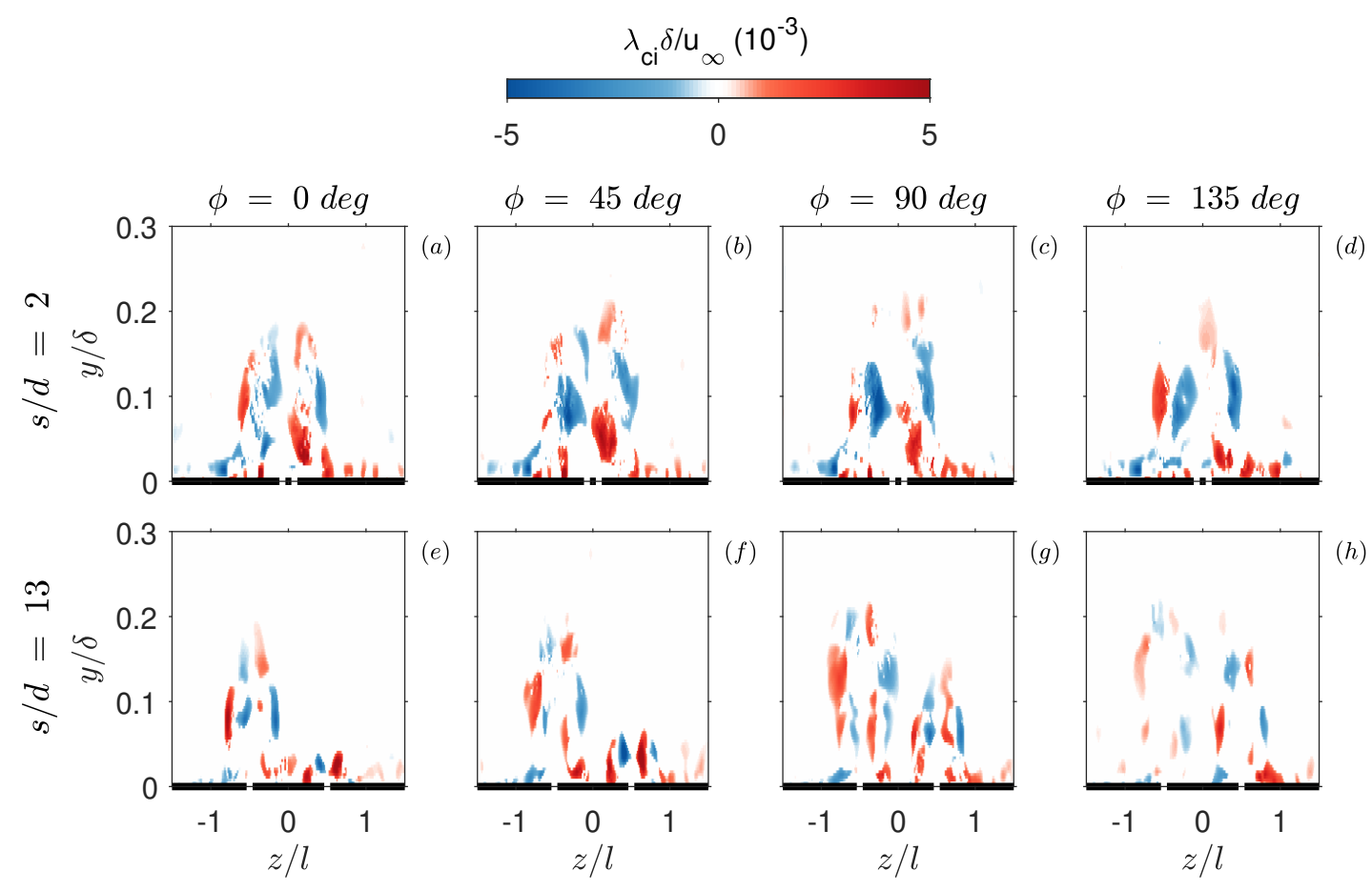

FIG. 6 Vorticity-signed swirling strength contour plots demonstrating the influence of orifice spacing on the vectoring behaviour of twin jets in crossflow for $s / d=2(\mathrm{a})-(\mathrm{d})$ and $s / d=13(\mathrm{e})-(\mathrm{h})$, all at $\beta$ of $78 \mathrm{deg}$ and $x / \delta=0$.

horizontal directions if a larger field of view was used. Eqn. 4 includes summations of all velocities within the area defined by the half-circle.

$$
\gamma=\arctan \left(\sum \Delta w / \sum \Delta v\right)
$$

For all cases at $x / \delta=0$, the half-line corresponding to $r / d=5$ is used to delimit the flowfield due to the low penetration of the jets while other downstream locations use a value of $r / d=10$ as this is more representative of the jet characteristics due to increased penetration. Comparison of the vectoring angle, $\gamma$, against downstream locations for various phase differences indicates that vectoring is minimal at $x / \delta=0$ (Fig. 7). Such observations contrast with the vectoring angles achieved by Berk et al. (2016) under quiescent conditions, where $\gamma$ was measured to be in the range of $25-45 \mathrm{deg}$ under similar actuation conditions for $s / d=2$. Values of $\gamma$ not exceeding $15 \mathrm{deg}$ can be attributed to the crossflow which introduces an adverse pressure gradient and causes the jets to be deflected in the streamwise direction, hence limiting the interaction between the jets in the near-field. In fact, further downstream, vectoring values similar to Berk et al. (2016) can be recovered as there has been adequate interaction between the jets and the vectoring mechanism involving attraction and impact has been achieved. The presence of the crossflow introduces a temporal and spatial delay in the interaction between the two jets. Additionally, analysis of the yawing offset provides an insight into the behaviour of vectoring with downstream location. Apart from being inclined, Fig. 7 also reveals that the structures experience a horizontal shift with respect to the center-line. The yawing offset is maximum at $x / \delta$ of 1 , beyond which this value stays constant. This potentially indicates that vectoring effect is only significant in the near-field region of the jet and the existence of a threshold streamwise location where the influence of the crossflow overpowers the vectoring mechanism.

\section{Turbulent kinetic energy}

The degree of interaction of twin jets in a crossflow can also be reflected in the analysis of the flowfield unsteadiness. Following Van Buren et al. (2014), the integrated turbulent kinetic energy is used as a measure of the overall flow unsteadiness at a given streamwise location. The turbulent kinetic energy is computed by integrating the velocity fluctuations over the region of interest and normalising with the mean blowing velocity of the jets.

$$
\Phi_{T K E}=\frac{1}{2} \int \frac{u^{\prime 2}+v^{\prime 2}+w^{\prime 2}}{\bar{u}_{j}^{2}} d z d y .
$$

From Fig. 8, a peak in unsteadiness appears downstream of the orifice exit, before the normalised turbulent kinetic energy decays and reaches a moderately constant value. These maxima in turbulent kinetic energy 


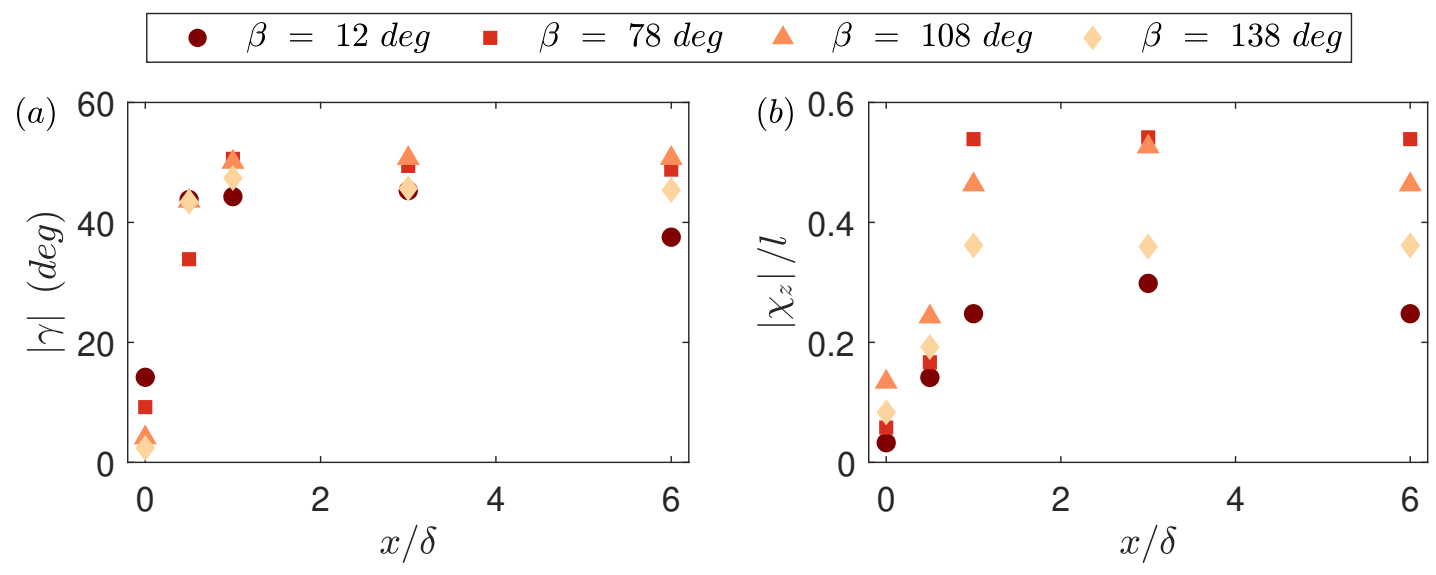

FIG. 7 Variation of (a) vectoring angle and (b) yawing offset, with streamwise location for differing phase difference between the twin jets.

correspond to the coherent structures generated by the synthetic jet actuators in the vicinity of the orifices. The location of these peaks displays singular sensitivity to the spanwise separation distance between the orifices. An increase in $s / d$ from 2 to 6.5 causes the peak turbulent kinetic energy to shift from $x / \delta$ of 0.5 to 1 . The location of this peak also indicates the point at which the interaction between the structures emanating from the two jets is at its apogee. Higher turbulent kinetic energy values for $s / d$ of 2 and 6.5 , as opposed to 13 , also attest the additional unsteadiness arising from the interaction of twin jets. Furthermore, for $s / d$ of 2 , the streamwise location of the peak turbulent kinetic energy and optimum vectoring angle and yawing offset values are coincident. This confirms that the vectoring behaviour is prominent only up to this downstream location and the influence of the crossflow in driving the jets become increasingly prevalent as the streamwise distance from the orifice increases, substantiated by the reduction in turbulent kinetic energy to a stable value which is representative of the steady features of the flowfield.

\section{Shear stresses}

One of the key features when using synthetic jet actuators in a drag reduction framework is to target large scale structures in the flowfield. Consequently, there is a reorganisation of the turbulence in the boundary layer. Such an effect can be analysed by computing the stresses derived from triple decomposition of the three component velocity vector in the cross plane (Eqn. 6) (Medjnoun et al. 2020).

$$
u_{i}(y, z, t)=U(y)+\tilde{u}(y, z)+u^{\prime}(y, z, t)
$$

where $u_{i}$ is the instantaneous velocity field at a given streamwise location, $U$ is the spatial and time-averaged velocity profile, $\tilde{u}$ is the spatial deviation field and $u^{\prime}$ is the time-dependent velocity fluctuations, part of the Reynolds double decomposition.

The triple decomposition allows derivation and quantification of the shear stresses (dispersive, turbulent and total) which arise from the mean flow. Dispersive shear stress arises from the double averaging and is important in the transport of momentum flux, which impacts the organisation of structures within the turbulent boundary layer. The diffusion mechanism for the transport of momentum is caused by eddies across planes of mean shear parallel to the wall. The turbulent shear stress is related to the subsequent velocity fluctuations in time which result in associated momentum fluctuations.

The sensitivity of the total shear stress on the phase difference between the jets and vectoring behaviour is assessed by computing the spanwise average of the total shear stress and plotting the subsequent variation with wall-normal distance (Fig. 9).

$$
<\overline{\tau_{x y}}>=\nu \frac{\partial<U>}{\partial y}-\left(<\overline{u^{\prime} v^{\prime}}>+<\overline{\tilde{u} \tilde{v}}>\right),
$$

where $\langle$.$\rangle denotes a spanwise averaged term, \overline{\tau_{x y}}$ is the total shear stress, $\nu \frac{\partial\langle U\rangle}{\partial y}$ is the viscous shear stress, $\overline{u^{\prime} v^{\prime}}$ is the turbulent shear stress and $\overline{\tilde{u}} \tilde{v}$ is the dispersive shear stress. Although, the total shear stress is linked to the skin-friction drag close to the wall, the reflection of the laser sheet off the surface makes the data points at the wall unreliable and hence direct measure of skin-friction drag cannot be inferred from the total shear stress values computed in this study. Fig. 9 corroborates earlier observations and indicates that the vectoring angle, $\beta$, only influences the total shear stress for the smallest orifice spacing. In the case of $s / d$ of 6.5 and 13 , the spanwise and time average profiles are similar and follow the same trend with streamwise location. Hence, any further discussion on the variation of total shear stress with phase difference will focus on $s / d=2$. 


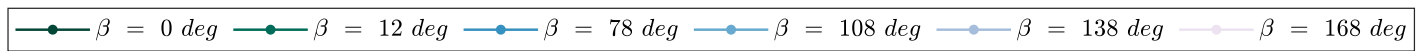

(a)

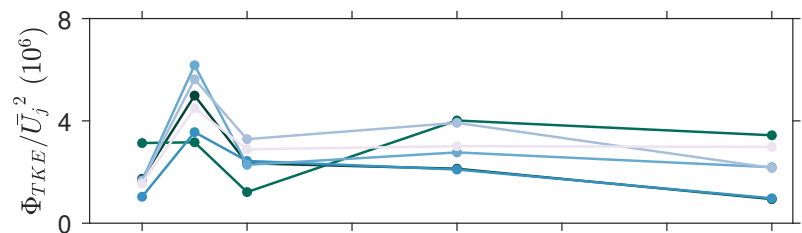

(b)

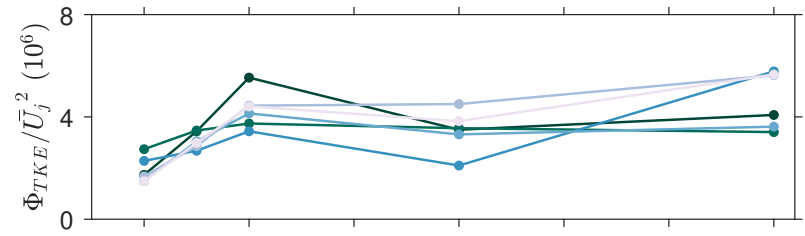

$(c)$

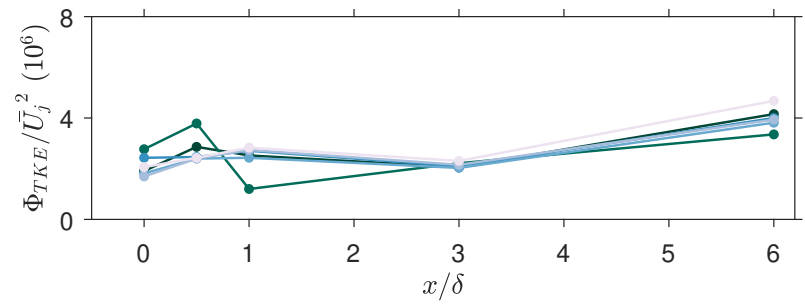

FIG. 8 Variation of the normalised intergrated turbulent kinetic energy with streamwise location as the phase difference between the jets is varied for (a) $s / d=2$, (b) $s / d=6.5$ and (c) $s / d=13$.

Fig. 9 also reveals that the maximum total shear stress is achieved when the twin jets are at a phase difference of $78 \mathrm{deg}$ for $x / \delta$ greater than 0.5 . The primary component of the total shear stress at these locations is the dispersive shear stress. Thus, it is sensible to assume that the transport of momentum, when in a twin jet configuration is maximum when the jets are roughly at a phase difference of $78 \mathrm{deg}$. When actuated under these conditions, the leading jet is at the peak blowing stage while the lagging jet is still at the onset of blowing. Due to the influence of the crossflow, the vortical structures emanating from the leading jet already convect downstream before the lagging jet reaches peak blowing. Although vectoring is induced, there is limited interaction between the jets and the vortical structures from both jets remain coherent throughout the actuation cycle resulting in optimised momentum transport.

In fact, the flowfield of twin jets in this configuration and under these actuation parameters is analogous to having a single jet at twice the actuation frequency, $f$. However, $2 f$ in this case corresponds to $800 \mathrm{~Hz}$ and at such a high frequency, synthesising and forming a single jet might not be possible. Hence, the use of twin jets enables circumvention of such a limitation. Furthermore, the wall-normal location of the maximum total shear stress increases with $x / \delta$. Although such behaviour is expected as the depth of penetration of the resulting jet increases with streamwise distance, this demonstrates that profiles of the total shear stress provide an alternate method to quantify and predict the trajectory of jets in a boundary layer.

These results also provide an insight in the setup of SJAs in an array for optimum performance in relevant applications. From a separation control or drag reduction perspective, having equally spaced single synthetic jet actuators in an array does not necessarily correspond to an optimum configuration. It can be inferred from the findings that twin jet configurations with $s / d$ less than 6.5 can be more efficient at momentum transport and generate improved flow control properties. Rather than a conventional configuration of linearly spaced single SJAs, an array should consist of linearly spaced pair of actuators. Such an adaptation would not only simplify the conditions necessary for synthesising synthetic jets but also provide benefits in terms of shear stress and skin-friction drag reduction, which still has not been fully explored.

\section{CONCLUSION}

This study investigates the interaction between two parallel synthetic jets when influenced by a crossflow. For same shape and size orifices, the separation distance and phase difference between the jets are varied while actuation parameters such as the mean blowing velocity and frequency are kept constant. Both synthetic jets are issued in a turbulent boundary layer whose freestream velocity is maintained at $10 \mathrm{~m} / \mathrm{s}$ throughout. The main conclusions stemming from these results are: 


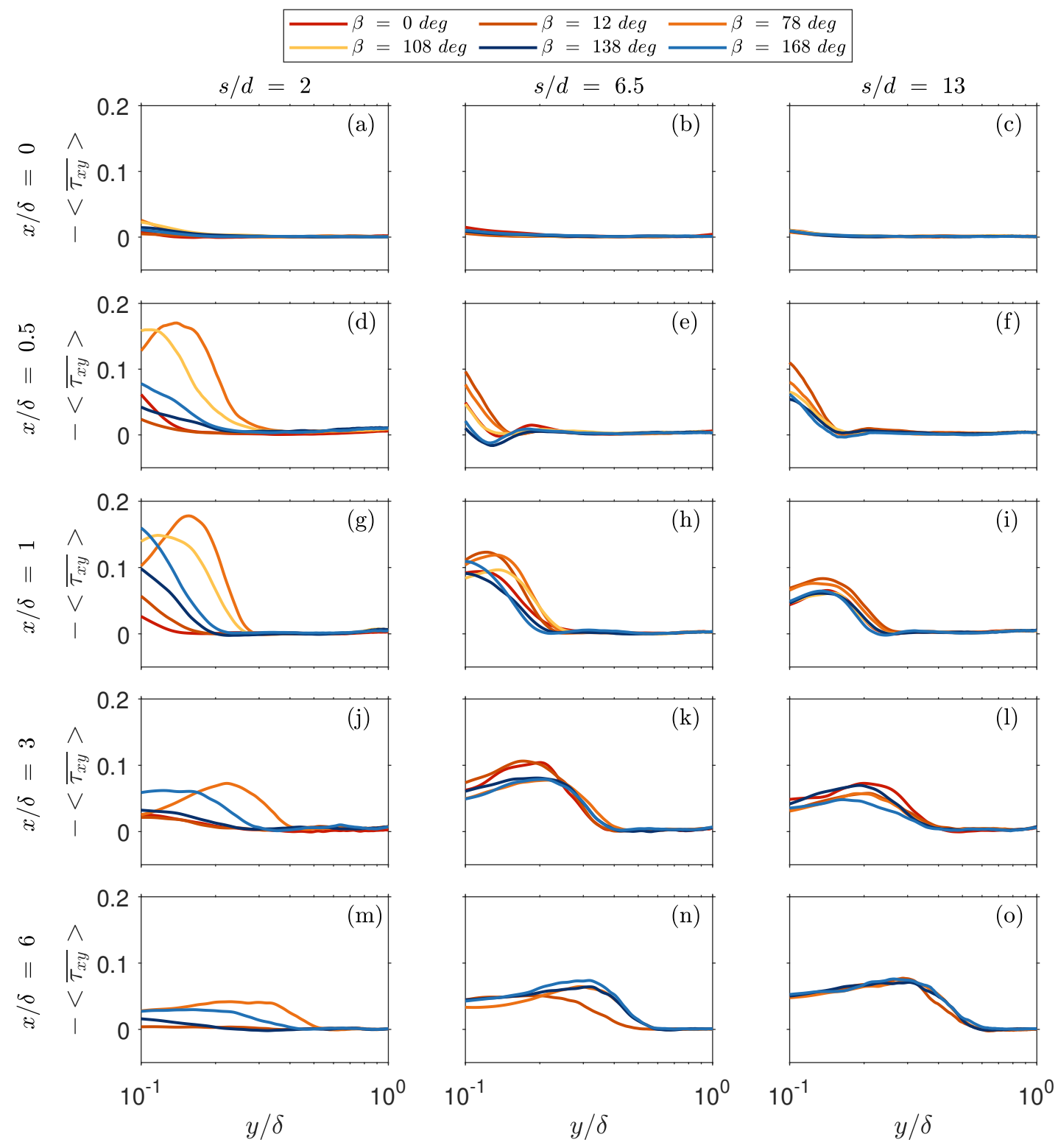

FIG. 9 Variation of the spanwise and time averaged total shear stress as the phase difference and orifice spacing between the twin jets is changed.

- Interaction between twin jets under the influence of a crossflow only occurs for separation distances, $s / d$, less than 6.5. These results corroborate the findings of Watson et al. (2003) and McGuinn et al. (2016) under quiescent conditions.

- Vectoring angles similar to quiescent flow conditions can be recovered at a streamwise location of $x / \delta=0.5$ for $s / d=2$. The crossflow is believed to only induce a temporal and spatial delay in the interaction between the two jets.

- A phase difference of 78 deg has the highest total shear stress, which consists primarily of the dispersive shear stress, and has a flowfield which is analogous to a single jet at twice the actuation frequency. These results provide an alternative method of reaching optimised momentum transfer within the turbulent boundary layer by combining two parallel jets under specific actuation conditions.

- These findings can be useful for synthetic jet array configurations used in flow separation control applications in order to maximise the performance.

Finally, this study addresses the shortcomings of multiple rectangular parallel jets in crossflow. The parametric investigation on the influence of orifice spacing and phase difference provides a deeper understanding of the simi- 
larities and differences in the behaviour and interaction of twin jets, when subjected to a crossflow as opposed to quiescent flow. These findings could pave the way for arrays of actuators to be configured in varied ways for optimised flow control and drag reduction applications.

\section{ACKNOWLEDGMENTS}

The authors acknowledge the financial support from the Engineering and Physical Sciences Research Council (ESPRC Grant No. EP/L006383/1) and Airbus. All data supporting this study are openly available from the University of Southampton repository at https ://doi.org/XX.XXXX/SOTON /XXXXX.

\section{REFERENCES}

Beresh, Steven J, Justin L. Wagner, and Barton L. Smith (2016), "Self-calibration performance in stereoscopic PIV acquired in a transonic wind tunnel," Exp. Fluids 57 (4), 1-17.

Berk, Tim, and Bharathram Ganapathisubramani (2019), "Effects of vortex-induced velocity on the development of a synthetic jet issuing into a turbulent boundary layer," J. Fluid Mech. 870, 651-679.

Berk, Tim, Guillaume Gomit, and Bharathram Ganapathisubramani (2016), "Vectoring of parallel synthetic jets: A parametric study," J. Fluid Mech. 804, 467-489.

Bhattacharya, Sayantan, John J Charonko, and Pavlos P Vlachos (2017), "Stereo-particle image velocimetry uncertainty quantification," Meas. Sci. Technol. 28 (1), 015301.

Ganapathisubramani, B, E. K. Longmire, and I. Marusic (2002), "Investigation of three dimensionality in the near field of a round jet using stereo PIV," J. Turbul. 3, 10.1088/1468-5248/3/1/016

Glezer, Ari, and Michael Amitay (2002), "Synthetic Jets," Annu. Rev. Fluid Mech. 34 (1), 503-529

Hasnain, Zohaib, Alison B. Flatau, James E. Hubbard, and Rahul Mulinti (2012), "Numerical study and experimental validation of the interaction of multiple synthetic jet actuators with cross flow," 50th AIAA Aerosp. Sci. Meet. Incl. New Horizons Forum Aerosp. Expo. (January), 1-16.

Holdeman, James D (1993), "Mixing of multiple jets with a confined subsonic crossflow," Prog. Energy Combust. Sci. 19 (1), 31-70

Holman, Ryan, Yogen Utturkar, Rajat Mittal, Barton L. Smith, and Louis Cattafesta (2005), "Formation Criterion for Synthetic Jets," AIAA J. 43 (10), 2110-2116.

Honami, Shinji, and Masahiro Motosuke (2012), "Vortex interaction of in-line synthetic jets injected at different phase in low reynolds number cross flow," 6th AIAA Flow Control Conf. 2012 (June), 1-8.

Jabbal, M, and S. Zhong (2008), "The near wall effect of synthetic jets in a boundary layer," Int. J. Heat Fluid Flow 29 (1), 119-130.

Jabbal, Mark, and Shan Zhong (2010), "Particle image velocimetry measurements of the interaction of synthetic jets with a zero-pressure gradient laminar boundary layer," Phys. Fluids 22 (6), 063603

Jankee, Girish K, and Bharathram Ganapathisubramani (2020), "Comparison between object and image plane cross-correlation for stereoscopic PIV in the presence of pixel locking," Exp. Fluids 61 (3), 89

Jankee, Girish K, and Bharathram Ganapathisubramani (2021), "Scalings for rectangular synthetic jet trajectory in a turbulent boundary layer," Journal of Fluid Mechanics 915, A57.

Luo, Zhen Bing, and Zhi Xun Xia (2008), "PIV measurements and mechanisms of adjacent synthetic jets interactions," Chinese Phys. Lett. 25 (2), 612-615.

McGuinn, Alan, Daniel I. Rylatt, and Tadhg S. O'Donovan (2016), "Heat transfer enhancement to an array of synthetic air jets by an induced crossflow," Appl. Therm. Eng. 103, 996-1003

Medjnoun, T, C. Vanderwel, and B. Ganapathisubramani (2020), "Effects of heterogeneous surface geometry on secondary flows in turbulent boundary layers," J. Fluid Mech. 886, $10.1017 / \mathrm{jfm} .2019 .1014$.

Orkwis, Paul D, and Claudio Filz (2003), "Characterization of dual two dimensional synthetic jets in cross flow at low Mach number," 33rd AIAA Fluid Dyn. Conf. Exhib. (June), 1-14.

Schlüter, J U, and T Schönfeld (2000), "LES of jets in cross flow and its application," Flow, Turbul. Combust. 65, 177203.

Smith, Barton L, and Ari Glezer (1998), "The formation and evolution of synthetic jets," Phys. Fluids 10 (9), 2281-2297

Smith, Barton L, and Ari Glezer (2005), "Vectoring of Adjacent Synthetic Jets," AIAA J. 43 (10), 2117-2124

Smith, Douglas R (2002), "Interaction of a Synthetic Jet with a Crossflow Boundary Layer," AIAA J. 40 (11), 2277-2288.

Toy, N, E. Savory, S. McCusker, and P. J. Disimile (1993), "The interaction region associated with twin jets and a normal crossflow," in AGARD,Computational Exp. Assess. Jets Cross Flow.

Van Buren, Tyler, Michael Beyar, Chia Min Leong, and Michael Amitay (2016a), "Three-dimensional interaction of a finite-span synthetic jet in a crossflow," Phys. Fluids 28 (3), 037105

Van Buren, Tyler, Chia Min Leong, Edward Whalen, and Michael Amitay (2016b), "Impact of orifice orientation on a finite-span synthetic jet interaction with a crossflow," Phys. Fluids 28 (3), 037106 .

Van Buren, Tyler, Edward Whalen, and Michael Amitay (2014), "Vortex formation of a finite-span synthetic jet: High Reynolds numbers," Phys. Fluids 26 (1), 014101.

Watson, Mark, Artur J. Jaworski, and Norman J. Wood (2003), "Contribution to the understanding of flow interactions between multiple synthetic jets," AIAA J. 41 (4), 747-749

Wen, Xin, Hui Tang, and Fei Duan (2015), "Vortex dynamics of in-line twin synthetic jets in a laminar boundary layer," Phys. Fluids 27 (8), 083601

Wen, Xin, Hui Tang, and Fei Duan (2016), "Interaction of inline twin synthetic jets with a separated flow," Phys. Fluids 28 (4), 043602.

Yu, Daeyoung, M. S. Ali, and Joseph H. W. Lee (2006), "Multiple Tandem Jets in Cross-Flow," J. Hydraul. Eng. 132 (9), 971-982.

Zang, B, and T. H. New (2017), "Near-field dynamics of parallel twin jets in cross-flow," Phys. Fluids 29 (3), 035103 